\title{
Efeitos da espécie vegetal exótica invasora Elaeis guineensis Jacq. sobre a fauna de formigas em uma Floresta Estacional Semidecidual em regeneração
}

\section{Effects of the invasive exotic plant species Elaeis guineensis Jacq. on ant fauna in a regenerating seasonal semi-deciduous forest}

\author{
1 Rita de Kassia Muniz-Araujo kassia12araujo@hotmail.com \\ 2 Elaine Bernini \\ ${ }^{3}$ Frederico Lage-Pinto
}

\footnotetext{
1 Bacharela em Ecologia. Laboratório de Ecologia Costeira e Oceânica da Universidade Federal da Paraíba, Campus IV. Universidade Federal da Paraíba (UFPB).

2 Doutora em Ecologia e Recursos Naturais. Campus IV. Universidade Federal da Paraíba (UFPB).
}

3 Doutor em Ecologia e Recursos Naturais. Campus IV. Universidade Federal da Paraíba (UFPB).

\section{Resumo}

As formigas representam um dos principais grupos de insetos utilizados em estudos que avaliam a qualidade ambiental. 0 objetivo deste estudo foi avaliar se a presença de Elaeis guineensis Jacq. (dendê) afeta a fauna de formigas em uma Floresta Estacional Semidecidual em regeneração, na REBIO Guaribas, Estado da Paraíba. As coletas foram realizadas por meio de armadilhas de queda. Para caracterização ambiental, foram realizadas medidas de temperatura, umidade, luminosidade, cobertura e espessura da serapilheira e parâmetros fitossociológicos. Registramos um total de 1.418 espécimes de formigas pertencentes a 51 espécies, 29 gêneros e sete subfamílias. Myrmicinae, Ponerinae e Formicinae foram as subfamílias com maior riqueza de espécies. Na área de Dendê, as espécies/morfoespécies com dominância $>50 \%$ foram Solenopsis sp.3, Nylanderia sp.1, Dinoponera quadriceps Kempf, 1971, Pheidole sp.2 e Wasmania auropunctata Roger, 1863 , enquanto na Mata foram Solenopsis sp.3, Pheidole sp.2, Nylanderia sp.1 e Pheidole sp.5. Em geral, a dominância dessas espécies indica que as áreas analisadas se encontram sob pressão antrópica. $A$ área de Dendê exibiu maiores valores de abundância, riqueza e diversidade de espécies, entretanto a invasão de E. guineensis nessa área está favorecendo W. auropunctata, que é considerada oportunista agressiva e pode deslocar a mirmecofauna nativa.

\section{Palavras-chave:}

Mata Atlântica. Mirmecofauna. Dendê.

\begin{abstract}
Ants represent one of the main groups of insects used in studies that aim to assess environmental quality. The aim of this study was to evaluate whether the presence of Elaeis guineensis Jacq. (palm oil) affects the ant fauna in a seasonal Semi-deciduous Forest in regeneration in REBIO Guaribas, State of Paraíba. Collections were carried out using pitfall traps. For environmental characterization, measurements of temperature, humidity, luminosity, cover and thickness of the litterfall and phytosociological parameters were performed. We recorded a total of 1,418 ant specimens belonging to 51 species, 29 genera and 7 subfamilies. Myrmicinae, Ponerinae and Formicinae were the subfamilies with the highest species richness. In the Dendê area, the species/ morphospecies with dominance greater than $50 \%$ were Solenopsis sp.3, Nylanderia sp.1, Dinoponera quadriceps Kempf, 1971, Pheidole sp.2 and Wasmania auropunctata Roger, 1863, while in the Forest, Solenopsis sp.3, Pheidole sp.2, Nylanderia sp.1 and Pheidole sp.5. In general, the dominance of these species indicates that the analyzed areas are under anthropic pressure. The Dendê area showed higher values of abundance, richness and diversity of species, however, the invasion of $E$. guineensis in this area is favoring W. auropunctata, which is considered an aggressive opportunist and can displace the native myrmecofauna.
\end{abstract}

\section{Keywords:}

Palm Oil. Atlantic Forest. Myrmecofauna.

Como você deve citar?

MUNIZ-ARAUJO, Rita de Kassia; BERNINI, Elaine; LAGE-PINTO, Frederico. Efeitos da espécie vegetal exótica invasora Elaeis guineensis Jacq. sobre a fauna de formigas em uma Floresta Estacional Semidecidual em regeneração. Cadernos UniFOA, Volta Redonda (RJ), v. 16, n. 46, p. 1-16, ago, 2021. 


\section{INTRODUÇÃO}

As formigas formam um dos grupos de insetos mais abundantes do planeta, estando presentes em praticamente todos os ambientes terrestres (HÖLLDOBLER e WILSON, 1990; BACCARO et al., 2015). Apesar disso, elas não se encontram distribuídas de forma homogênea, pois alguns fatores, como geografia, geologia e clima são responsáveis por definir sua persistência nos ambientes (FISHER, 2010).

A mirmecofauna exibe ampla distribuição e biomassa e é responsável por desempenhar diversas funções importantes nos ecossistemas (HÖLLDOBLER e WILSON, 1990; FISHER et al., 2000; HOFFMANN e ANDERSEN, 2003; WILSON e HÖLLDOBLER, 2005; ORTIZ et al., 2021). Além disso, possui considerável sensibilidade a distúrbios (HOFFMANN e ANDERSEN, 2003) e representa um dos principais grupos de insetos utilizados em estudos que visam avaliar a qualidade ambiental (KING et al., 1998; LOBRY DE LRUYN, 1999; UNDERWOOD e FISHER, 2006; UEHARA-PRADO et al., 2009; RIBAS et al., 2012; JIMÉNEZCARMONA et al., 2020).

Plantas exóticas invasoras em ambientes naturais ou a conversão dessas áreas em monoculturas são exemplos de impactos que geram consideráveis alterações sobre a mirmecofauna. Nesse sentido, estudos demonstram declínio na riqueza de formigas após a conversão de florestas naturais em plantações de eucalipto (MENTONE et al., 2011), de cana-de-açúcar (SANTOS et al., 2012) e de dendê (Elaeis guineensis Jacq.; BRÜHL; ELTZ, 2010). Além disso, a ocorrência de formigas invasoras geralmente é favorecida em plantações de seringueira (HOSOISHI et al., 2013), de eucalipto (MENTONE et al., 2011) e de dendê (ULLOA-CHACÓN e CHERIX, 1990).

Devido aos seus diversos usos, as plantações de dendê se tornaram uma das monoculturas que mais têm se expandido ultimamente, sendo cultivadas em mais de 43 países na região tropical (VIJAY et al., 2016). Além da introdução intencional por causa do cultivo, o dendê pode ser introduzido de forma acidental e acabar se dispersando e colonizando ambientes naturais. Essa espécie invasora adentra em fragmentos florestais, forma adensamentos e, em muitos casos, acaba se sobressaindo em relação a espécies nativas (LEÃO et al., 2011). Isso faz do dendê uma das principais espécies de plantas invasoras encontradas em Unidades de Conservação brasileiras (SAMPAIO e SCHMIDT, 2013), como é o caso da Reserva Biológica Guaribas (REBIO Guaribas).

A REBIO Guaribas constitui um dos últimos remanescentes de Floresta Atlântica do Estado da Paraíba e abriga espécies raras, endêmicas e ameaçadas de extinção (ICMBIO, 2003). Essa Unidade de Conservação é dividida em três fragmentos (SEMA I, II e III), sendo composta por Floresta Estacional Semidecidual e Mata de Tabuleiro. Todos os fragmentos da floresta encontram-se em regeneração natural após cortes para extração de madeira e carvão. Apesar de o Plano de Manejo da REBIO Guaribas prever a remoção de espécies exóticas invasoras, foram observadas áreas com alta densidade de plântulas, indivíduos jovens e árvores de dendê mesclando-se com espécies vegetais nativas.

O fato de o dendê estar invadindo Unidades de Conservação realça a importância de se entender o seu efeito na estrutura e funcionamento dos ecossistemas para haver o planejamento adequado de sua remoção. $O$ objetivo deste estudo foi avaliar se a presença do dendê afeta a fauna de formigas em uma Floresta Estacional Semidecidual na REBIO Guaribas.

\section{MATERIAL E MÉTODOS}

O estudo foi realizado em uma Floresta Estacional Semidecidual localizada em um dos fragmentos da Reserva Biológica Guaribas (SEMA III), no município de Rio Tinto, estado da Paraíba, Brasil. A SEMA 
III possui 339 ha (ICMBIO, 2003), sendo, aproximadamente, $5 \%$ cobertos por árvores de dendê. 0 entorno do fragmento é ocupado por plantações de cana-de-açúcar, propriedades rurais e área urbana. 0 clima predominante na região é quente e úmido com temperaturas médias anuais em torno de $24^{\circ} \mathrm{C}$ e $26^{\circ} \mathrm{C}$, segundo a classificação de Köppen. A precipitação anual varia entre 1.750 e $2.000 \mathrm{~mm}$ com as maiores médias ocorrendo entre fevereiro e julho e, as menores, entre outubro e dezembro (ICMBIO, 2003).

Duas áreas foram selecionadas no local de estudo, uma com ocorrência de dendê, doravante denominada Dendê, e uma área com ausência dessa espécie, doravante denominada Mata. As áreas foram distanciadas em $60 \mathrm{~m}$ e localizadas a cerca de $200 \mathrm{~m}$ da borda da reserva ( $06^{\circ} 48^{\prime} 28^{\prime \prime} \mathrm{S}$ e $\left.35^{\circ} 05^{\prime} 14^{\prime \prime} \mathrm{O}\right)$. Em cada área, foram estabelecidas duas transeções paralelas de $50 \mathrm{~m}$ cada, distanciadas em $10 \mathrm{~m}$. Ao longo de cada transeção, foram distribuídas seis armadilhas de queda do tipo pitfall (distanciadas em $10 \mathrm{~m}$ ), totalizando 12 por área. Os pitfalls com capacidade de $750 \mathrm{~mL}$ foram preenchidos com 250 $\mathrm{mL}$ de uma mistura de água e detergente neutro e permaneceram em campo por 48 horas. Após esse período, o conteúdo do recipiente foi filtrado e transferido para potes de plástico contendo álcool $92 \%$. Em seguida, o material foi encaminhado ao laboratório para triagem, montagem e identificação. As coletas foram realizadas no fim do período chuvoso (agosto de 2018). As formigas foram identificadas em nível de espécie/morfoespécie de acordo com Baccaro et al. (2015) e literatura especializada. 0 material coletado foi depositado no Laboratório de Ecologia Costeira e Oceânica da Universidade Federal da Paraíba (Campus IV, Rio Tinto).

Ao lado de cada pitfall, foram realizadas medidas de temperatura do ar, umidade, luminosidade, cobertura do solo e espessura da serapilheira. A coleta de dados foi realizada entre 8 e 11 horas da manhã, utilizando-se termo-higrômetro digital para medir temperatura e umidade, e luxímetro digital portátil, para medir os níveis de luminosidade. Todas as medidas foram realizadas ao nível do solo por, pelo menos, 1 min ou até estabilização das medidas. A cobertura da serapilheira foi estimada visualmente dentro de quadrantes $(50 \times 50 \mathrm{~cm}$ ), sendo realizadas três medidas aleatórias no entorno de cada ponto de amostragem ( $1 \mathrm{~m}$ de distância). A espessura da serapilheira foi mensurada dentro do quadrante com auxílio de uma régua graduada. Para a caracterização geral da estrutura da vegetação, foi empregado o método ponto quadrante nos pontos correspondentes à instalação dos pitfalls. Os indivíduos vivos com diâmetro $\geq 5 \mathrm{~cm}$ tiveram a altura medida e foram classificados como dendê ou outra espécie arbórea.

Para avaliar a diversidade de formigas, foram utilizados o índice de Shannon $\left(H^{\prime}\right)$ e o índice de Simpson (D). A uniformidade de distribuição dos indivíduos foi avaliada pelo índice de equitabilidade de Pielou (J') (MAGURRAN, 2004).

Para efeito de abundância, foram calculadas as frequências absoluta e relativa. A frequência absoluta das espécies foi calculada através da fórmula:

$$
F A i=\frac{N P .100}{N P T}(1)
$$

Onde: NP é o número de pitfalls em que a espécie "i" ocorreu e NPT corresponde ao número total de pitfalls amostrados. 
As frequências relativas foram calculadas através da fórmula:

$$
F(\%)=\frac{F i}{F t} .100(2)
$$

Onde: $F_{i}$ corresponde ao número de registros de cada espécie e $F_{t}$ o número de registros totais de espécies de cada área.

A suficiência amostral foi avaliada através de reamostragem por permutação (1.000 aleatorizações; GOTELLI e COLLWEL, 2001). A riqueza foi estimada através de dois estimadores: Jackknife 2, por permitir a realização de uma estimativa confiável da riqueza mesmo com baixa amostragem (ZAHL, 1977) e Chao 1, por ser um estimador simples e poderoso que se baseia na abundância das espécies raras (CHAO, 1984). As análises foram realizadas com auxílio do software $R$ (R DEVELOPMENT CORE TEAM, 2019), utilizando-se o pacote vegan (OKSANEN et al., 2019).

Para identificar espécies indicadoras, foi utilizado o método do Valor Indicador Individual (IndVal; DUFRÊNE; LEGENDRE, 1997). De acordo com Mcgeoch et al. (2002), esse método combina o grau de especificidade de uma determinada espécie para um status ecológico, por exemplo, tipo de habitat e sua fidelidade dentro do status, medida através da sua percentagem de ocorrência.

A temperatura, umidade, luminosidade, espessura da serapilheira e cobertura do solo foram comparadas por meio do teste $t$ ou teste $\mathrm{U}$ (quando as premissas de normalidade e homocedasticidade de variâncias não foram atendidas). Os parâmetros fitossociológicos (densidade relativa e altura) foram calculados no programa FITOPAC ${ }^{\circledR}$ versão 2.1 (SHEPHERD, 2010).

\section{RESULTADOS}

Foram amostrados um total de 1.418 espécimes pertencentes a 51 espécies, 29 gêneros e sete subfamílias de formigas (Tabela 1). Nas áreas de Dendê e de Mata, foram coletados 989 e 429 espécimes, respectivamente. As subfamílias que apresentaram maior riqueza de espécies em ordem decrescente foram Myrmicinae, Ponerinae e Formicinae, abrangendo 91\% das espécies coletadas (Figura 1).

Os gêneros com maior riqueza de espécies foram Pheidole (10 espécies), Solenopsis (quatro espécies), Camponotus, Pseudomyrmex e Odontomachus (três espécies cada). No Dendê, cinco espécies exibiram frequência absoluta maior que $50 \%$ na seguinte ordem: Solenopsis sp. 3 > Nylanderia sp.1 > Dinoponera quadriceps Kempf, 1971 = Pheidole sp.2 = Wasmania auropunctata Roger, 1863 (Tabela 1). $\mathrm{Na}$ Mata, quatro espécies mostraram frequência absoluta maior que 50\%: Solenopsis $\mathrm{sp} .3>$ Pheidole sp. 2 > Nylanderia sp. 1 = Pheidole sp.5. A frequência relativa das espécies apresentou a mesma ordem da frequência absoluta em cada área (Tabela 1). 
Figura 1 - Frequência relativa das subfamílias amostradas, nas áreas de Dendê e de Mata, analisadas na Reserva Biológica Guaribas, Rio Tinto, Brasil.

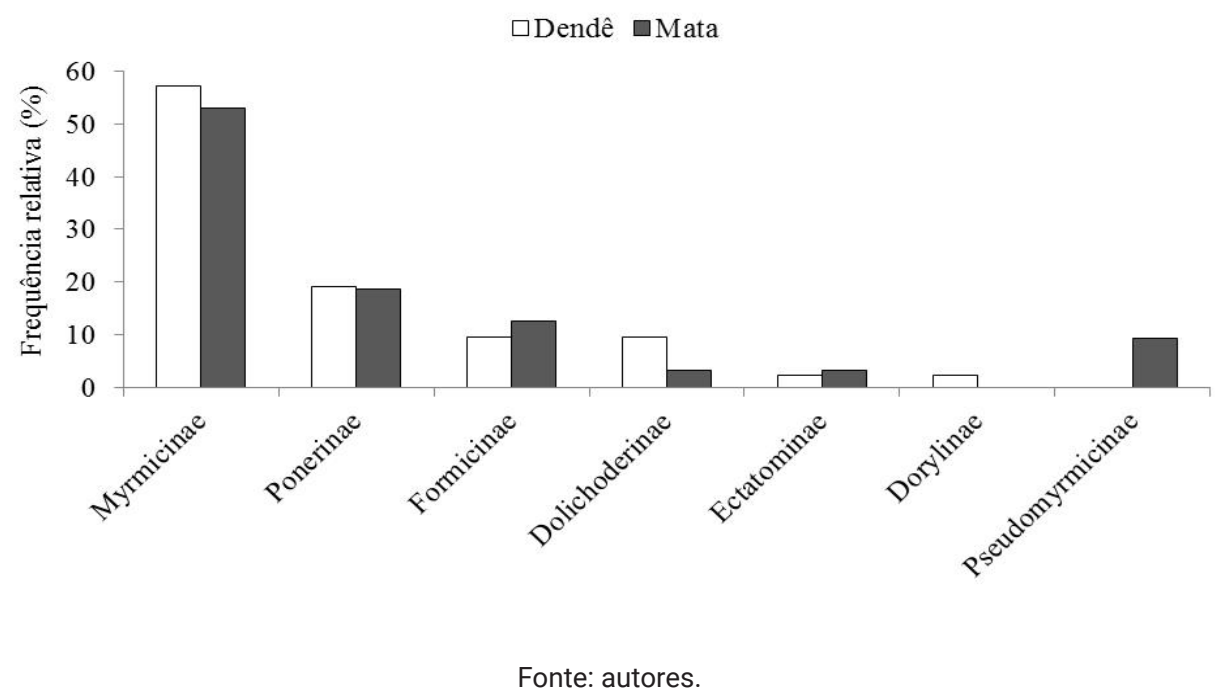

A área de Dendê exibiu maiores valores de abundância, riqueza de gêneros e diversidade de Shannon, enquanto a diversidade de Simpson e a equitabilidade foram similares entre as áreas (Tabela 2). A curva de acumulação de espécies não mostrou tendência de estabilização em uma assíntota. 0 estimador Jackknife 2 estimou 66 e 50 espécies para a área de Dendê e Mata, respectivamente. 0 estimador Chao 1 estimou 61 espécies para a área de Dendê e 45 espécies para a área de Mata (Figura 2). Apenas W. auropunctata (63\%) e $D$. quadriceps (52\%) demonstraram níveis significativos de indicação $(\mathrm{p} \leq 0,05)$, ambas indicadoras da área de Dendê.

A umidade e luminosidade foram significativamente maiores na área de Dendê, enquanto a cobertura do solo e a espessura da serapilheira foram superiores na área de Mata. A temperatura do ar não diferiu entre as áreas (Tabela 3 ). Houve ausência de E. guineensis na área de Mata, mas, na área de Dendê, essa espécie apresentou $56,3 \%$ de densidade relativa (Tabela 4). A altura das árvores foi similar entre as áreas (Tabela 4). 
Efeitos da espécie vegetal exótica invasora Elaeis guineensis Jacq. sobre a fauna de formigas em uma Floresta Estacional Semidecidual em regeneração

Figura 2 - Curvas médias de acumulação de espécies de formigas, intervalos de confiança de 95\% e estimadores de riqueza, para as áreas de Dendê (linha e tracejados em preto) e de Mata (linha e tracejados em vermelho), analisadas na Reserva Biológica Guaribas, Rio Tinto, Brasil.

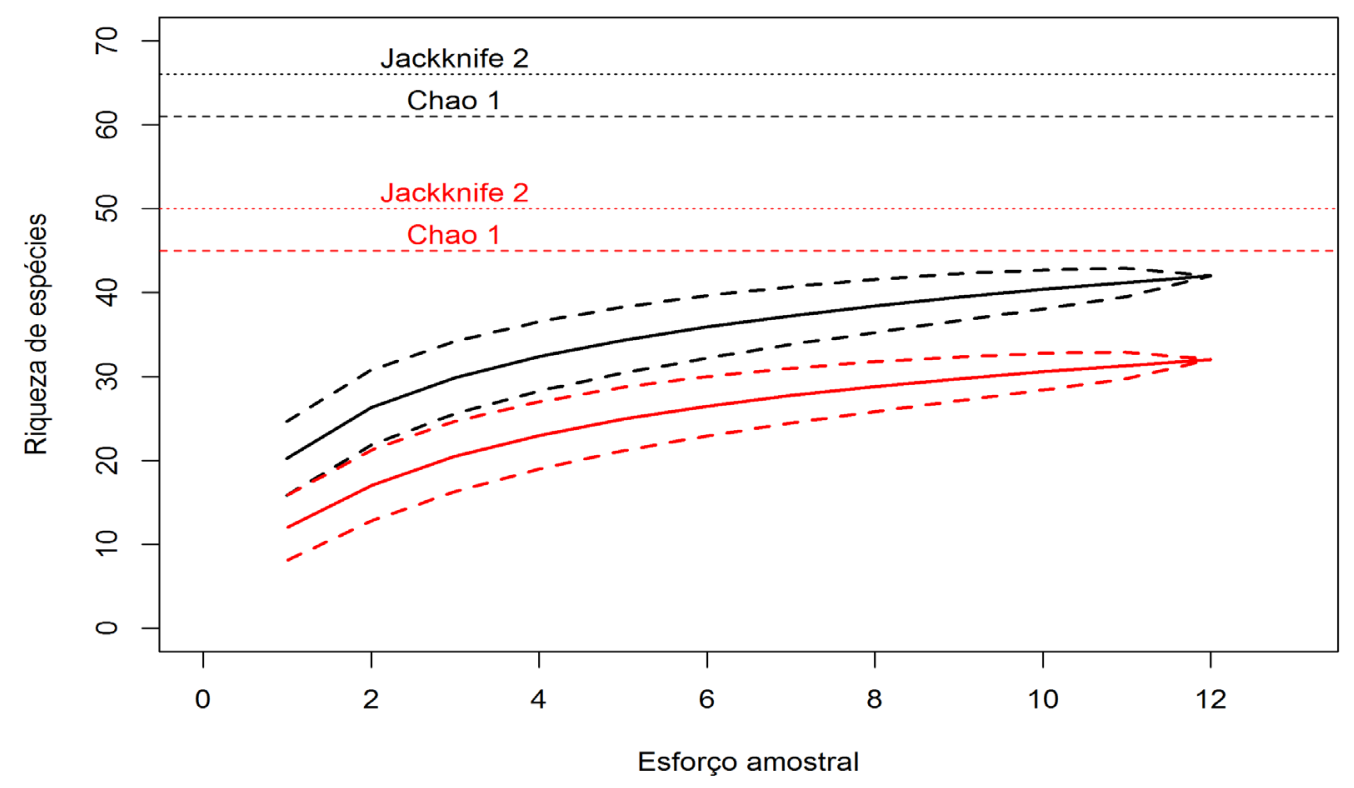

Fonte: autores. 
Tabela 1- Registros de ocorrência (RO), frequência absoluta (FA) e frequência relativa (FR) das espécies de formigas nas áreas analisadas na Reserva Biológica Guaribas, Rio Tinto, Brasil. N = 12.

\begin{tabular}{|c|c|c|c|c|c|c|}
\hline \multirow{2}{*}{ Táxon } & \multicolumn{3}{|c|}{ Dendê } & \multicolumn{3}{|c|}{ Mata } \\
\hline & RO & FA (\%) & FR (\%) & RO & FA (\%) & FR (\%) \\
\hline \multicolumn{7}{|l|}{ SUBFAMÍLIA DOLICHODERINAE } \\
\hline \multicolumn{7}{|l|}{ Tribo Dolichoderini } \\
\hline Azteca sp.1 & 3 & 25,0 & 2,5 & 2 & 16,7 & 2,3 \\
\hline Azteca sp.2 & 1 & 8,3 & 0,8 & - & - & - \\
\hline Dolichoderus imitator Emery, 1894 & 1 & 8,3 & 0,8 & - & - & - \\
\hline Tapinoma sp.1 & 1 & 8,3 & 0,8 & - & - & - \\
\hline \multicolumn{7}{|l|}{ SUBFAMÍLIA DORYLINAE } \\
\hline Labidus sp. 1 & 3 & 25 & 2,5 & - & - & - \\
\hline \multicolumn{7}{|l|}{ SUBFAMÍLIA ECTATOMMINAE } \\
\hline \multicolumn{7}{|l|}{ Tribo Ectatommini } \\
\hline Gnamptogenys horni Santschi, 1929 & 2 & 16,7 & 1,7 & 1 & 8,3 & 1,1 \\
\hline \multicolumn{7}{|l|}{ SUBFAMÍLIA FORMICINAE } \\
\hline \multicolumn{7}{|l|}{ Tribo Camponotini } \\
\hline Camponotus sp. 1 & 1 & 8,3 & 0,8 & 1 & 8,3 & 1,1 \\
\hline Camponotus sp.2 & 1 & 8,3 & 0,8 & - & - & - \\
\hline Camponotus sp. 3 & - & - & - & 1 & 8,3 & 1,1 \\
\hline \multicolumn{7}{|l|}{ Tribo Plagiolepidini } \\
\hline Brachymyrmex sp.1 & 4 & 33,3 & 3,3 & 1 & 8,3 & 1,1 \\
\hline Nylanderia sp.1 & 9 & 75,0 & 7,4 & 7 & 58,3 & 8,0 \\
\hline \multicolumn{7}{|l|}{ SUBFAMÍLIA MYRMICINAE } \\
\hline \multicolumn{7}{|l|}{ Tribo Attini } \\
\hline Acanthognathus sp. 1 & 1 & 8,3 & 0,8 & - & - & - \\
\hline Atta sexdens & - & - & - & 3 & 25,0 & 3,4 \\
\hline Cephalotes atratus Linnaeus, 1758 & - & - & - & 2 & 16,7 & 2,3 \\
\hline Mycetophylax lilloanus Kusnezov, 1949 & 1 & 8,3 & 0,8 & 2 & 16,7 & 2,3 \\
\hline Mycocepurus goeldii Forel, 1893 & 1 & 8,3 & 0,8 & - & - & - \\
\hline Myrmicocrypta sp.1 & 1 & 8,3 & 0,8 & - & - & - \\
\hline Pheidole sp.1 & 4 & 33,3 & 3,3 & - & - & - \\
\hline Pheidole sp.2 & 8 & 66,7 & 6,6 & 9 & 75,0 & 10,3 \\
\hline Pheidole sp.3 & 1 & 8,3 & 0,8 & - & - & - \\
\hline Pheidole sp.4 & 3 & 25,0 & 2,5 & 3 & 25,0 & 3,4 \\
\hline Pheidole sp.5 & 4 & 33,3 & 3,3 & 7 & 58,3 & 8 \\
\hline Pheidole sp.6 & 3 & 25,0 & 2,5 & 4 & 33,3 & 4,6 \\
\hline Pheidole sp.7 & 2 & 16,7 & 1,7 & 1 & 8,3 & 1,1 \\
\hline Pheidole sp.8 & 1 & 8,3 & 0,8 & 2 & 16,7 & 2,3 \\
\hline Pheidole sp.9 & 2 & 16,7 & 1,7 & - & - & - \\
\hline Pheidole sp.10 & 1 & 8,3 & 0,8 & 1 & 8,3 & 1,1 \\
\hline Sericomyrmex sp.1 & 2 & 16,7 & 1,7 & 4 & 33,3 & 4,6 \\
\hline Strumigenys denticulata Mayr, 1887 & 4 & 33,3 & 3,3 & 3 & 25,0 & 3,4 \\
\hline Strumigenys elongata Roger, 1863 & 2 & 16,7 & 1,7 & - & - & - \\
\hline Wasmannia auropunctata Roger, 1863 & 8 & 66,7 & 6,6 & 1 & 8,3 & 1,1 \\
\hline
\end{tabular}


Efeitos da espécie vegetal exótica invasora Elaeis guineensis Jacq. sobre a fauna de formigas em uma Floresta Estacional Semidecidual em regeneração

\begin{tabular}{|c|c|c|c|c|c|c|}
\hline Tribo Crematogastrini & & & & & & \\
\hline Carebara reticulata Fernández, 2004 & 2 & 16,7 & 1,7 & - & - & - \\
\hline Crematogaster sp.1 & 1 & 8,3 & 0,8 & 1 & 8,3 & 1,1 \\
\hline \multicolumn{7}{|l|}{ Tribo Solenopsidini } \\
\hline Megalomyrmex sp.1 & - & - & - & 1 & 8,3 & 1,1 \\
\hline Solenopsis sp. 1 & 2 & 16,7 & 1,7 & - & - & - \\
\hline Solenopsis sp.2 & 6 & 50,0 & 5,0 & 2 & 16,7 & 2,3 \\
\hline Solenopsis sp. 3 & 10 & 83,3 & 8,3 & 10 & 83,3 & 11,5 \\
\hline Solenopsis sp.4 & 1 & 8,3 & 0,8 & - & - & - \\
\hline \multicolumn{7}{|l|}{ SUBFAMÍLIA PONERINAE } \\
\hline \multicolumn{7}{|l|}{ Tribo Ponerini } \\
\hline Dinoponera quadriceps Kempf, 1971 & 8 & 66,7 & 6,6 & 3 & 25,0 & 3,4 \\
\hline Hypoponera opacior Forel, 1893 & 4 & 33,3 & 3,3 & 3 & 25,0 & 3,4 \\
\hline Mayaponera constricta Mayr, 1884 & 3 & 25,0 & 2,5 & 2 & 16,7 & 2,3 \\
\hline Neoponera apicalis Latreille, 1802 & 2 & 16,7 & 1,7 & - & - & - \\
\hline Neoponera villosa Fabricius, 1804 & - & - & - & 1 & 8,3 & 1,1 \\
\hline Odontomachus haematodus Linnaeus, 1758 & 1 & 8,3 & 0,8 & - & - & - \\
\hline Odontomachus sp.2 & 1 & 8,3 & 0,8 & - & - & - \\
\hline Odontomachus sp.3 & 1 & 8,3 & 0,8 & 4 & 33,3 & 4,6 \\
\hline Rasopone sp.1 & 4 & 33,3 & 3,3 & - & - & - \\
\hline Rasopone sp.2 & - & - & - & 1 & 8,3 & 1,1 \\
\hline \multicolumn{7}{|l|}{ SUBFAMÍLIA PSEUDOMYRMECINAE } \\
\hline \multicolumn{7}{|l|}{ Tribo Pseudomyrmecini } \\
\hline Pseudomyrmex oculatus Smith, 1855 & - & - & - & 2 & 16,7 & 2,3 \\
\hline Pseudomyrmex gracilis Fabricius, 1804 & - & - & - & 1 & 8,3 & 1,1 \\
\hline Pseudomyrmex sp.3 & - & - & - & 1 & 8,3 & 1,1 \\
\hline Total $=$ & 121 & - & 100 & 87 & - & 100 \\
\hline
\end{tabular}

Fonte: autores.

Tabela 2 - Análise faunística de Formicidae nas áreas analisadas na Reserva Biológica Guaribas, Rio Tinto, Brasil, onde: N: Número total de indivíduos; S: Riqueza de gêneros; S': Riqueza de espécies; $H^{\prime}$ : Índice de diversidade de Shannon; $D$ : Índice de diversidade de Simpson; e J': Índice de equitabilidade de Pielou.

\begin{tabular}{ccccccc}
\hline Área & N & S & $\mathbf{S}^{\prime}$ & $\boldsymbol{H}^{\prime}$ & $\boldsymbol{D}$ & $\boldsymbol{J}^{\prime}$ \\
\hline Dendê & 989 & 25 & 42 & 3,38 & 0,95 & 0,91 \\
\hline Mata & 429 & 22 & 32 & 3,17 & 0,94 & 0,92 \\
\hline
\end{tabular}

Fonte: autores. 
Tabela 3 - Variáveis ambientais das áreas analisadas na Reserva Biológica Guaribas, Rio Tinto, Brasil. MD: Média; DP: desvio-padrão; Med: mediana; e CV: coeficiente de variação. Nas colunas, letras distintas indicam diferença significativa entre as áreas $(p \leq 0,05)$. * Teste $t$; ** Teste $U$.

\begin{tabular}{|c|c|c|c|c|c|c|}
\hline Área & & $\begin{array}{l}\text { Temperatura } \\
\left({ }^{\circ} \mathrm{C}\right)^{\star}\end{array}$ & Umidade (\%)* & Luminosidade (Lux) $^{\star \star}$ & $\begin{array}{l}\text { Espessura da } \\
\text { serapilheira } \\
(\mathrm{cm})^{\star}\end{array}$ & $\begin{array}{c}\text { Cobertura do } \\
\text { solo }(\%)^{\star}\end{array}$ \\
\hline \multirow{3}{*}{ Dendê } & $M D \pm D P$ & $29,2 \pm 2,7 a$ & $59,4 \pm 4,0 \mathrm{a}$ & $4804,8 \pm 14049,2 \mathrm{a}$ & $5,5 \pm 1,7 b$ & $90,1 \pm 8,9 b$ \\
\hline & Med & 28,4 & 59,0 & 272,5 & 4,8 & 92,2 \\
\hline & CV & 9,2 & 6,7 & 292,4 & 31,4 & 9,9 \\
\hline \multirow{3}{*}{ Mata } & $\mathrm{MD} \pm \mathrm{DP}$ & $29,8 \pm 0,4 a$ & $53,3 \pm 3,1 b$ & $199,3 \pm 37,6 b$ & $6,9 \pm 2,0 \mathrm{a}$ & $99,7 \pm 0,5 \mathrm{a}$ \\
\hline & Med & 29,8 & 54,0 & 182,0 & 6,5 & 99,9 \\
\hline & CV & 1,3 & 5,8 & 18,9 & 29,4 & 0,5 \\
\hline
\end{tabular}

Fonte: autores.

Tabela 4 - Densidade relativa e altura dos indivíduos arbóreos nas áreas analisadas na Reserva Biológica Guaribas, Rio Tinto, Brasil. NR: indivíduos não registrados.

\begin{tabular}{cccc}
\hline Área & Espécies & Densidade relativa $(\%)$ & Altura $(\mathbf{m})$ \\
\hline \multirow{2}{*}{ Dendê } & Elaeis guineensis & 56,3 & 11,5 \\
\cline { 2 - 4 } & Outras & 43,7 & 12,3 \\
\hline \multirow{2}{*}{ Mata } & Elaeis guineensis & NR & NR \\
\cline { 2 - 4 } & Outras & 100 & 11,9 \\
\hline & & \multirow{2}{*}{ Fonte: autores. }
\end{tabular}

\section{DISCUSSÃO}

Myrmicinae é uma subfamília caracterizada por formigas que exibem hábitos diversificados de alimentação e nidificação (HÖLLDOBLER e WILSON, 1990). Isso explica sua maior representatividade no presente estudo, conforme observado em diversos ecossistemas brasileiros (DELABIE et al., 2006; CARDOSO et al., 2010; FREIRE et al., 2012; CANTARELLI et al., 2015; TAVARES, 2017; JORY e FEITOSA, 2020; SOUZA-CAMPANA, 2020). A composição da mirmecofauna foi diferenciada entre as áreas analisadas, pois, das 51 espécies registradas, apenas 23 foram compartilhadas, destacando-se Solenopsis sp.3, Nylanderia sp. 1 e Pheidole sp.2.

Em habitats perturbados, são esperadas distribuições de frequência relativa com uma proporção maior de espécies mais dominantes (HILL e HAMER, 1998) e isso foi observado tanto na área de Mata quanto na área de Dendê. A dominância de espécies dos gêneros Solenopsis, Pheidole e Nylanderia reflete as mudanças na estrutura da vegetação (RESENDE et al., 2011; SUGUITURU et al., 2013) e indicam que as áreas analisadas se encontram sob pressão antrópica. De fato, essas áreas apresentam uma matriz de entorno composta por plantações e área urbana. $O$ fragmento de floresta estudado passa por processo de regeneração natural desde a década de 1940, mas a extração de madeira era recorrente, mesmo após a criação da REBIO Guaribas, em 1990 (ICMBio, 2003). Atualmente, o corte seletivo foi drasticamente reduzido, mas a caça no interior do fragmento e incêndios ainda são registrados no entorno da Unidade de Conservação. 
Efeitos da espécie vegetal exótica invasora Elaeis guineensis Jacq. sobre a fauna de formigas em uma Floresta Estacional Semidecidual em regeneração

Solenopsis, Pheidole e Nylanderia são gêneros caracterizados por espécies oportunistas e generalistas (DELABIE e FOWLER, 1995; SILVESTRE e SILVA, 2001; RESENDE et al., 2011), que não encontram restrições para nidificar e para obter alimento em áreas antropizadas, porque apresentam características ecológicas e biológicas que favorecem a exploração do ambiente (FOWLER et al., 1994; LUTINSKI et al., 2014). Formigas dominantes constroem ninhos grandes, possuem colônias extremamente populosas e apresentam alta territorialidade (DEJEAN e CORBARA, 2003). Solenopsis e Nylanderia estão entre os gêneros que apresentam espécies de formigas mais agressivas e são elencadas como potencialmente invasoras e responsáveis por deslocar espécies nativas (DELABIE e FOWLER, 1995; BACCARO et al., 2015).

As espécies W. auropunctata e $D$. quadriceps foram bioindicadoras da área de Dendê. W. auropunctata é oportunista com alta capacidade de ocupar ambientes perturbados, pois apresenta elevada agressividade interespecífica e habilidade em explorar uma variedade de recursos alimentares (ARMBRECHT e ULLOA-CHACÓN, 2003; SOUZA et al., 2008). Essa espécie é capaz de deslocar a mirmecofauna local, podendo se tornar dominante (ULLOA-CHACÓN e CHERIX, 1990). A maior frequência de W. auropunctata na área de Dendê, em relação à Mata, provavelmente responde à menor diversidade de espécies vegetais, devido à elevada densidade de $E$. guineensis. Outros estudos têm demonstrado o potencial de domínio dessa espécie (ARMBRECHT e ULLOA-CHACÓN, 2003).

Dinoponera quadriceps é encontrada na Mata Atlântica, Cerrado e Caatinga, no Nordeste (KEMPF, 1971; PAIVA e BRANDÃO, 1995). Essa espécie é onívora, com dieta composta por matéria orgânica morta (principalmente artrópodes), frutos e presas vivas (ARAÚJO e RODRIGUES, 2006). Dinoponera quadriceps é mais propensa a forragear em ambientes mais úmidos (MEDEIROS et al., 2014) e isso explicaria sua maior dominância na área de Dendê que exibiu umidade significativamente maior do que a Mata.

Houve maior riqueza e diversidade de formigas na área de Dendê em relação à Mata, contrariando vários estudos que documentaram reduções na riqueza de espécies de formigas do solo em plantações de dendê, em comparação com florestas nativas (TAYLOR, 1977; PFEIFFER, 2008; BRÜHL; ELTZ, 2010; FAYLE et al., 2010). Alterações no microclima e redução na disponibilidade de locais para nidificação têm sido apontadas como responsáveis pela perda de riqueza de formigas do solo em monoculturas de dendê (BRÜHL e ELTZ, 2010). Isso se deve ao fato de que essas plantações apresentam solo desprotegido, com condições mais secas e quentes, que afetam o desempenho e a distribuição da mirmecofauna (TORRES, 1984). Além disso, a camada de serapilheira é quase ausente nessas áreas, prejudicando espécies que nidificam (CARVALHO e VASCONCELOS, 1999). Outro fator que também pode influenciar a perda de riqueza de espécies em monoculturas de dendê é o decréscimo da diversidade de espécies vegetais, que reduz a heterogeneidade ambiental e disponibilidade de recursos (RIBAS et al., 2003; TEWS et al., 2004).

No presente estudo, a temperatura não diferiu entre as áreas e, embora a espessura e a cobertura de serapilheira tenham sido maiores na Mata, não houve ausência de serapilheira na área de Dendê. Nessa área, a floresta mostrou-se estruturalmente mais homogênea, por apresentar mais de 50\% de contribuição de E. guineensis, mas ainda possui espécies vegetais nativas. Esses resultados evidenciam as diferenças da área de Dendê em relação às monoculturas analisadas em outros estudos, com ambientes mais homogêneos e sujeitos a drásticas alterações microclimáticas em relação às florestas nativas. Fayle et al. (2010) não encontraram diferenças na riqueza de formigas em epífitas sobre árvores de dendê e espécies nativas, devido à ausência de alterações microclimáticas e disponibilidade de locais para nidificação. Conforme observado no presente estudo, uma maior riqueza de formigas em ambientes mais homogêneos em termos de composição florística já foi documentada (MARINHO et al., 2002; MIRANDA et al., 2013).

A maior umidade registrada na área de Dendê pode ser um dos fatores que promoveu maior riqueza e diversidade de espécies. Nessa área, também foram observados valores significativamente 
superiores de luminosidade, que podem favorecer algumas espécies oportunistas e/ou generalistas (ANDERSEN, 2019). Além disso, a camada de serapilheira na área de Dendê é constituída por componentes de espécies vegetais nativas e por palha e frutos de dendê que, aliado à maior umidade, pode estar permitindo o estabelecimento de maior número de espécies no solo, devido ao incremento de recursos e locais para nidificação. A disponibilidade de recursos também pode estar sendo incrementada por fezes de marsupiais e roedores, pois frutos das espécies vegetais exóticas invasoras $E$. guineensis e Syzygium cumini (L.) Skeels são mais atrativos do que aqueles de espécies nativas para essa fauna na REBIO Guaribas (SILVA, 2013).

Considerando a invasão do dendê na floresta como um distúrbio moderado, nossos resultados corroboram a hipótese do distúrbio intermediário, que sugere que a diversidade é maior em níveis intermediários de frequência, intensidade e duração de uma perturbação (CONNELL, 1978). Nesse sentido, ambientes naturais moderadamente perturbados podem oferecer condições intermediárias que favorecem o estabelecimento de formigas (LUKE et al., 2014), que, no presente estudo, são relacionadas à maior disponibilidade de recursos e locais para nidificação. Por fim, é importante destacar que, embora a área de Dendê apresente maior riqueza e diversidade de formigas, a invasão de $E$. guineensis está favorecendo $W$. auropunctata, considerada uma espécie oportunista agressiva mesmo dentro de sua faixa natural de ocorrência (LE BRETON et al., 2014) e pode deslocar a mirmecofauna nativa (ULLOACHACÓN e CHERIX, 1990).

\section{AGRADECIMENTOS}

À Me. Marília Maria Silva da Costa, pelo auxílio na identificação das formigas. À UFPB, Campus IV, pelo apoio referente à infraestrutura e ao transporte para atividades de campo. À REBIO Guaribas, pelo apoio à pesquisa.

\section{REFERÊNCIAS}

ANDERSEN, A. Responses of ant communities to disturbance: five principles for understanding the disturbance dynamics of a globally dominant faunal group. Journal of Animal Ecology, London, v. 88, p. 350-362, 2019.

ARAÚJO, A.; RODRIGUES, Z. Foraging Behavior of the queenless ant Dinoponera quadriceps Santschi (Hymenoptera: Formicidae). Neotropical Entomology, Londrina, v. 35, n. 2, p. 159-164, 2006.

ARMBRECHT, I.; ULLOA-CHACÓN, P. The little fire ant Wasmannia auropunctata (Roger) (Hymenoptera: Formicidae) as a diversity indicator of ants in tropical dry forest fragments of Colombia. Environmental Entomology, Lanham, v. 32, n. 3, p. 542-547, 2003.

BACCARO, F. B.; FEITOSA, R. M.; FERNANDEZ, F.; IZZO, T. J.; SOUZA, J. L. P.; SOLAR, R. Guia para gêneros de formigas do Brasil. Manaus: INPA, 2015. $388 \mathrm{p}$.

BRÜHL, C. A.; ELTZ, T. Fuelling the biodiversity crisis: species loss of ground-dwelling forest ants in oil palm plantations in Sabah, Malaysia (Borneo). Biodiversity and Conservation, New York, v, 19, p. 519-529, 2010.

CANTARELLI, E. B.; FLECK, M. D.; GRANZOTTO, F.; CORASSA, J. N.; D’AVILA, M. Diversidade de formigas (Hymenoptera: Formicidae) da serrapilheira em diferentes sistemas de uso do solo. Ciência Florestal, Santa Maria, v. 25, n.3, p. 607-616, 2015. 
CARDOSO, D. C.; SOBRINHO, T. G.; SCHOEREDER, J. H. Ant community composition and its relationship with phytophysiognomies in a Brazilian Restinga. Insectes Sociaux, Basel, v. 57, p. 293-301, 2010.

CARVALHO, K. S.; VASCONCELOS, H. L. Forest fragmentation in central Amazonia and its effects on litter-dwelling ants. Biological Conservation, Boston, v. 91, n. 2-3, p. 151-157, 1999.

CASTILHO, G. A. Estrutura da fauna de formigas epígeas em fragmentos de Floresta Atlântica Semidecidual com diferentes tamanhos. 2013. 97 f. Dissertação (Mestrado em Biologia Animal) - Universidade Estadual Paulista "Júlio de Mesquita Filho", São José do Rio Preto, 2013.

CONNELL, J. H. Diversity in tropical rain forests and coral reefs: high diversity of trees and corals is maintained only in a no equilibrium state. Science, New York, v. 199, n. 4335, p. 1302-1310, 1978.

CHAO, A. Non-parametric estimation of number of classes in a population. Scandinavian Journal of Statistics, Oxford, v. 11, p. 265-270, 1984.

DEJEAN, A.; CORBARA, B. Review of mosaics of dominant ants in rainforests and plantations. In: BASSET, Y.; KITCHING, R.; MILLER, S.; NOVOTNY, V. (Ed.). Arthropods of tropical forests: spatio-temporal dynamics and resource use in the canopy. Cambridge: Cambridge University Press, 2003. p. 341-347.

DELABIE, J. H. C.; FOWLER, H. G. Soil and litter cryptic ant assemblages of Bahian cocoa plantations. Pedobiologia, München, v. 39, p. 423-433, 1995.

DELABIE, J. H. C.; PAIM, V. R. L. M.; NASCIMENTO, I. C.; CAMPIOLO, S.; MARIANO, C. S. F. As formigas como indicadores biológicos do impacto humano em manguezais da costa sudeste da Bahia. Neotropical Entomology, Londrina v. 35, n. 5, p. 602-615, 2006.

DUFRÊNE, M.; LEGENDRE, P. Species assemblages and indicator species: the need for a flexible asymmetrical approach. Ecological Monographs, Ithaca, v. 67, n. 3, p. 345-366, 1997.

FAYLE, T. M.; TURNER, E. C.; SNADDON, J. L.; DHEY, V. K.; CHUNG, A. Y. C.; EGGLETON, P.; FOSTER, W. A. Oil palm expansion into rain forest greatly reduces ant biodiversity in canopy, epiphytes and leaf litter. Basic and Applied Ecology, Berlin, v. 11, p. 337-345, 2010.

FISHER, B. L. Biogeography. In: LACH, L.; PARR, C. L.; ABBOTT, K. L. (Ed.). Ant ecology. New York: Oxford University Press, 2010. p. 18-37.

FISHER, B. L.; MALSCH, A. K. F.; GADAGKAR, R.; DELABIE, J. H. C.; VASCONCELOS, H. L.; MAJER, J. D. Applying the ALL Protocol: selected case studies. In: AGOSTI, D.; MAJER, J. D.; ALONSO, L. A.; SCHULTZ, T. R. (Ed.). Ants standard methods for measuring and monitoring biodiversity. Washington: Smithsonian Institution Press, 2000. p. 207-214.

FOWLER, H. G.; SCHLINDWEIN, M. N.; MEDEIROS, M. A. Exotic ants and community simplification in Brazil: a review of the impact of exotic ants on native ant assemblages. In: WILLIAMS, D. F. (Ed.). Exotic ants: biology, impact, and control of introduced species. Boulder: Westview Press, 1994. p. 151-162.

FREIRE, C. B.; OLIVEIRA, G. V.; MARTINS, F. R. S.; SOUZA, L. E. C.; RAMOS-LACAU, L. S.; CORREAA, M. M. Riqueza de formigas em áreas preservadas e em regeneração de caatinga arbustiva no sudoeste da Bahia, Brasil. Revista Brasileira de Biociências, Porto Alegre, v. 10, n. 1, p. 131-134, 2012. 
GOTELLI, N.; COLWELL, R. K. Quantifying biodiversity: procedures and pitfalls in the measurement and comparison of species richness. Ecology Letters, Montpellier, v. 4, n. 4, p. 379-391, 2001.

HILL, J. K.; HAMER, K. C. Using species abundance curves as indicators of habitat disturbance in tropical forests. Journal of Applied Ecology, London, v. 35, p. 458-460, 1998.

HOFFMANN, B. D.; ANDERSEN, A. N. Responses of ants to disturbance in Australia, with particular reference to functional groups. Austral Ecology, Alice Springs, v. 28, p. 444-464, 2003.

HÖLLDOBLER, B.; WILSON, E. O. The ants. Cambridge: Harvard University Press, 1990. 732 p.

HOSOISHI, S.; NGOC, A. L.; YAMANE, S.; OGATA, K. Ant diversity in rubber plantations (Hevea brasiliensis) of Cambodia. Asian Myrmecology, Tokyo, v. 5, p. 69-77, 2013.

ICMBIO - INSTITUTO CHICO MENDES DE CONSERVAÇÃO DA BIODIVERSIDADE. Plano de manejo da Reserva Biológica Guaribas. Brasília: ICMBio, 2003. 520 p.

JIMÉNEZ-CARMONA, F.; HEREDIA-ARÉVALO, A.M.; REYES-LÓPEZ, J.L. Ants (Hymenoptera: Formicidae) as an indicator group of human environmental impact in the riparian forests of the Guadalquivir river (Andalusia, Spain). Ecological Indicators, Amsterdam, v. 118, 106762, 2020.

JORY, T.T.; FEITOSA, R.M. First survey of the ants (Hymenoptera, Formicidae) of Piauí: filling a major knowledge gap about ant diversity in Brazil. Papéis Avulsos de Zoologia, São Paulo, v.60: e20206014, 2020.

KEMPF, W. W. A preliminary review of the ponerine ant genus Dinoponera Roger (Hymenoptera: Formicidae). Studia Entomologica, California, v. 14, n. 369-394, 1971.

KING, J. R.; ANDERSEN, A. N.; CUTTER, A. D. Ants as bioindicators of habitat disturbance: validation of the functional group model for Australia's humid tropics. Biodiversity and Conservation, New York, v. 7, p. $1627-1638,1998$.

LE BRETON, J.; DELABIE, J. H. C.; CHAZEAU, J.; DEJEAN, A.; JOURDAN H. Experimental evidence of large scale unicoloniality in the tramp ant Wasmannia auropunctata (Roger). Journal of Insect Behavior, New York, v. 17, p. 263-271, 2004.

LEÃO, T. C. C.; ALMEIDA, W. R.; DECHOUM, M.; ZILLER, S. R. Espécies exóticas invasoras no nordeste do Brasil: contextualização, manejo e políticas públicas. Recife: Cepan, 2011. 99 p.

LOBRY DE BRUYN, L. A. Ants as bioindicators of soil function in rural environments. Agriculture, Ecosystems \& Environment, Amsterdam, v. 74, p. 425-441, 1999.

LUKE, S. H.; FAYLE, T. M.; EGGLETON, P.; TURNER, E. C.; DAVIES, R. G. Functional structure of ant and termite assemblages in old growth forest, logged forest and oil palm plantation in Malaysian Borneo. Biodiversity and Conservation, New York, v. 23, p. 2817-2832, 2014.

LUTINSKI, J. A.; LUTINSKI, C. J.; CORTÊS, B. L.; MORAIS, A. B. Estrutura da comunidade de formigas (Hymenoptera: Formicidae) em quatro ambientes com diferentes níveis de perturbação antrópica. Ecología Austral, Buenos Aires, v. 24, n. 2, p. 229-237, 2014.

MAGURRAN, A. E. Measuring biological diversity. Oxford: Blackwell Science, 2004. 256 p. 
MARINHO, C. G. S.; ZANETTI, R.; DELABIE, J. H. C.; SCHILINDWEIN, M. N.; RAMOS, L. S. Diversidade de formigas (Hymenoptera: Formicidae) da serapilheira em eucaliptais (Myrtaceae) e área de Cerrado de Minas Gerais. Neotropical Entomology, Londrina, v. 3, n. 2, p. 187-195, 2002.

MCGEOCH, M.A.; RENSBURG, B.J.V.; BOTES, B. The verification and application of bioindicators: a case study of dung beetles in a savanna ecosystem. Journal of Applied Ecology, v.39, n.4, p.661-672, 2002.

MEDEIROS, J.; AZEVEDO, D. L. O.; SANTANA, M. L. A. D.; LOPES, T. R. P.; ARAÚJO, A. Foraging activity rhythms of Dinoponera quadriceps (Hymenoptera: Formicidae) in its natural environment. Journal of Insect Science, Madison, v. 14, n. 220, p. 1-9, 2014.

MENTONE, T. O.; DINIZ, E. A.; MUNHAE, C. B.; BUENO, O. C.; MORINI, M. S. C. Composição da fauna de formigas (Hymenoptera: Formicidae) de serapilheira em florestas semidecídua e de Eucalyptus spp., na região sudeste do Brasil. Biota Neotropica, Campinas, v. 11, n. 2, p. 237-246, 2011.

MIRANDA, T. A.; SANTANNA, A. S.; VARGAS, A. B.; ALMEIDA, F. S. Aspectos estruturais do ambiente e seus efeitos nas assembléias de formigas em ambientes de floresta e bosque. Cadernos UniFOA, Volta Redonda, v. 21, p. 63-72, 2013.

OKSANEN, J.; GUILLAUME, B. F.; FRIENDLY, M.; KINDT, R.; LEGENDRE, P.; MCGLINN, D.; MINCHIN, P. R.; O'HARA, R. B.; SIMPSON, G. L.; SOLYMOS, P.; STEVENS, M. H. H.; SZOECS, E.; WAGNER H. Vegan: community ecology package. 2019. R package version 2.5-4. Disponível em: https://CRAN.R-project.org/ package=vegan. Acesso em: 10 jan. 2021.

ORTIZ, D.P.; ELIZALDE, L.; PIRK, G.I. Role of ants as dispersers of native and exotic seeds in an understudied dryland. Ecological Entomology, Malden, 10.1111/een.13010, 2021.

PAIVA, R. V. S.; BRANDÃO, C. R. F. Nests, worker population, and reproductive status of workers, in the giant queenless ponerine ant Dinoponera Roger (Hymenoptera Formicidae). Ethology Ecology \& Evolution, Firenze, v. 7, p. 297-312, 1995.

PFEIFFER, M.; TUCK, H. C.; LAY, T. C. Exploring arboreal ant community composition and co-occurrence patterns in plantations of oil palm Elaeis guineensis in Borneo and Peninsular Malaysia. Ecography, Lund, v. 31, p. 21-32, 2008.

R CORE TEAM. R: a language and environment for statistical computing 2019. Disponível em: https:// www.R-project.org/. Acesso em: 10 jan. 2021.

RESENDE, J. J.; SANTOS, G. M. M.; NASCIMENTO, I. N.; DELABIE, J. H. C.; SILVA, E. M. S. Communities of ants (Hymenoptera: Formicidae) in different Atlantic Rain Forest phytophysionomies. Sociobiology, California, v. 58, p. 779-798, 2011.

RIBAS, C. R.; CAMPOS, R. B. F.; SCHMIDT, F. A.; SOLAR, R. R. C. Ants as indicators in Brazil: a review with suggestions to improve the use of ants in environmental monitoring programs. Psyche: A Journal of Entomology, New York, v. 2012, p. 1-23, 2012.

RIBAS, C. R.; SCHOEREDER, J. H.; PIC, M.; SOARES, S. M. Tree heterogeneity, resource availability, and larger scale processes regulating arboreal ant species richness. Austral Ecology, Alice Springs, v. 28, p. 305-314, 2003. 
SAMPAIO, A. B.; SCHMIDT, I. B. Espécies exóticas invasoras em unidades de conservação federais do Brasil. Biodiversidade Brasileira, Brasília, v. 3, n. 2, p. 32-49, 2013.

SANTOS, M. P. C. J.; CARRANO-MOREIRA, A. F.; TORRES, J. B. Diversidade de formigas epigéicas (Hymenoptera: Formicidae) em floresta ombrófla densa e em cultivo de cana-de açúcar, no município de Igarassu, PE. Revista Brasileira de Ciências Agrárias, Recife, v. 7, n. 4, p. 648-656, 2012.

SHEPHERD, G. J. FITOPAC versão 2.1. 2010. Disponível em: https://pedroeisenlohr.webnode.com.br/ fitopac. Acesso em: 06 ago. 2020.

SILVA, A. L. V. Remoção de frutos e sementes por roedores e marsupiais na Mata Atlântica da Paraíba. 2013. Monografia (Graduação em Biologia) - Universidade Federal da Paraíba, João Pessoa. 2013.

SILVESTRE, R.; SILVA, R. R. Guildas de formigas da Estação Ecológica Jataí, Luiz Antônio - SP - sugestões para aplicação do modelo de guildas como bio-indicadores ambientais. Biotemas, Florianópolis, v. 14, n. 1, p. 37-69, 2001.

SOUZA-CAMPANA, D.R.; CARVALHO, A.C.N.; CANALI, M.; SILVA, O.G.M.; MORINI, M.S.C.; FUJIHARA, R.T. A rapid survey of ground-dwelling ants (Hymenoptera: Formicidae) in an urban park from state of São Paulo, Brazil. Brazilian Journal of Biology, São Carlos, vol. 80, no. 3, p. 682-684, 2020.

SOUZA, E.; FOLLETT, P. A.; PRICE, D. K.; STACY, E. A. Field suppression of the invasive ant Wasmannia auropunctata (Hymenoptera: Formicidae) in a tropical fruit orchard in Hawaii. Journal of Economic Entomology, Riverside, v. 101, n. 4, p. 1068-1074, 2008.

SUGUITURU, S. S.; SOUZA, D. R.; MUNHAE, C. B.; PACHECO, R.; MORINI, M. S. C. Diversidade e riqueza de formigas (Hymenoptera: Formicidae) em remanescentes de Mata Atlântica na bacia hidrográfica do Alto Tietê, SP. Biota Neotropica, Campinas, v, 13, n. 2, p. 141-152, 2013.

TAVARES, B. L. P. Diversidade e composição da comunidade de formigas da Reserva Biológica Guaribas e suas aplicações para a conservação da Mata Atlântica no estado da Paraíba, Brasil. 2017. 89 f. Dissertação (Mestrado em Entomologia e Conservação da Biodiversidade) - Universidade Federal da Grande Dourados, Dourados. 2017.

TAYLOR, B. The ant mosaic on cocoa and other tree crops in Western Nigeria. Ecological Entomology, London, v. 2, p. 245-255, 1977.

TEWS, J.; BROSE, U.; GRIMM, V.; TIELBORGER, K.; WICHMANN, M. C.; SCHWAGER, M.; JETSCH, F. Animal species diversity driven by habitat heterogeneity/diversity: the importance of keystone structures. Journal of Biogeography, New York, v. 31, p. 79-92, 2004.

TORRES, J.A. Diversity and distribution of ant communities in Puerto Rico. Biotropica, Malden, v. 16, p. 296-303, 1984.

UEHARA-PRADO, M.; FERNANDES, J. O.; BELLO, A. M.; MACHADO, G.; SANTOS, A. J.; VAZ-De-MELLO, F. Z.; FREITAS, A. V. L. Selecting terrestrial arthropods as indicators of small-scale disturbance: a first approach in the Brazilian Atlantic Forest. Biological Conservation, Boston, v. 142, n. 6, p. 1220-1228, 2009. 
ULLOA-CHACÓN, P.; CHERIX, D. The little fire ant Wasmannia auropunctata R. (Hymenoptera: Formicidae). In: VANDER MEER, R. K.; JAFFEE, K.; CEDENO, A. (Ed.). Applied myrmecology: a world perspective. Boulder: Westview Press, 1990. p. 281-289.

UNDERWOOD, E. C.; FISHER B. L. The role of ants in conservation monitoring: if, when, and how. Biological Conservation, Boston, v. 132, n. 2, p. 166-182, 2006.

VIJAY, V.; PIMM, S. L.; JENKINS, C. N.; ZMITH, S. J. The impacts of oil palm on recent deforestation and biodiversity loss. Plos One, San Francisco, v. 11, n. 7, e0159668, 2016.

WILSON, E. O.; HÖLLDOBLER, B. The rise of the ants: a phylogenetic and ecological explanation. Proceedings of the National Academy of Sciences, Washington, v. 102, p. 7411-7414, 2005.

ZAHL S. Jackknifing an index of diversity. Ecology, New York, v. 58, p. 907-913, 1977. 